\title{
A eficácia da laserterapia no tratamento das radiodermatites: revisão integrativa
}

\author{
The effectiveness of laser therapy in the treatment of radiodermatitis: integrative review \\ La eficacia de la láserapia en el tratamiento de la radiodermatitis: revisión integrativa
}

Recebido: 26/01/2021 | Revisado: 02/02/2021 | Aceito: 03/02/2021 | Publicado: 09/02/2021

Esther Santos Lima

ORCID: https://orcid.org/0000-0001-6609-5258

Universidade Tiradentes, Brasil

E-mail: esther-lima092@outlook.com

Yasmin Gonçalves Costa

ORCID: https://orcid.org/0000-0002-8971-969X

Universidade Tiradentes, Brasil

E-mail: yasmingca2@gmail.com

Lohanne Neres dos Santos

ORCID: https://orcid.org/0000-0003-2474-0057

Universidade Tiradentes, Brasil

E-mail: lohanneneres@gmail.com

Victoria Souza Messias Santos

ORCID: https://orcid.org/0000-0002-2974-5861 Universidade Tiradentes, Brasil

E-mail: victoriasouza.mama@hotmail.com

Milena Guedes Cunha

ORCID: https://orcid.org/0000-0002-6643-8625 Universidade Tiradentes, Brasil

E-mail: milenaguedes2008@hotmail.com

Jéssica Lorrane Barreto Silva Santos

ORCID: https://orcid.org/0000-0001-9837-2539 Universidade Tiradentes, Brasil

E-mail: jesylorrane@hotmail.com

Queila Samara dos Santos Farias ORCID: https://orcid.org/0000-0002-4686-4539 Universidade Tiradentes, Brasil

E-mail: queilasamara20@gmail.com

Fernanda Costa Martins Gallotti

ORCID: https://orcid.org/0000-0002-9063-1273

Universidade Tiradentes, Brasil

E-mail: fernanda.gallotti@souunit.com.br

\begin{abstract}
Resumo
$\mathrm{O}$ presente trabalho tem como objetivo avaliar a eficácia no tratamento com terapia a laser em pacientes que desenvolveram dermatites induzidas pela radiação. A fim de atingir o objetivo proposto e tendo como caminho metodológico a revisão integrativa da literatura com abordagem qualitativa, foi realizada busca nas bases de dados SCIELO, PUBMED, LILACS e BDENF nos idiomas: português, inglês e espanhol, entre os anos de 2010 a 2020. Em $86 \%$ das pesquisas selecionadas para compor este artigo, houve resultados positivos em relação à eficácia do tratamento com a laserterapia, promovendo a reparação tecidual devido à redução da inflamação/dor e a indução da síntese de colágeno nas doses adequadas. Entretanto, outro estudo apontou boa eficácia com o PDL, porém observaram reações transitórias e do tratamento pós-laser como: dor, púrpura e eritema. Outros autores concluíram que aplicação do LED em pacientes antes da radioterapia apresentou melhoria na intensidade da dor devido à toxicidade da pele induzida por radiação. Conclui-se que a laserterapia demonstrou resultados satisfatórios no tratamento para lesão oncológica, constituindo uma alternativa valiosa no combate das radiodermatites.
\end{abstract}

Palavras-chave: Câncer; Laserterapia; Oncologia; Radiodermatites.

\begin{abstract}
The present work aims to evaluate the effectiveness of treatment with laser therapy in patients who have developed radiation-induced dermatitis. In order to achieve the proposed objective and using the integrative literature review with a qualitative approach as a methodological path, a search was performed in the SCIELO, PUBMED, LILACS and BDENF databases; in languages: Portuguese, English and Spanish, between the years 2010 to 2020. In $86 \%$ of the surveys selected to compose this article, there were positive results regarding the effectiveness of treatment with laser therapy, promoting tissue repair due to the reduction of inflammation / pain and induction of collagen synthesis at
\end{abstract}


appropriate doses. However, another study pointed to good efficacy with the PDL, but observed transient and post-laser treatment reactions such as: pain, purpura and erythema. Other authors concluded that application of LED in patients before radiotherapy showed an improvement in pain intensity due to radiation-induced skin toxicity. It is concluded that laser therapy has shown satisfactory results in the treatment for cancer lesions, constituting a valuable alternative in the fight against radiodermatitis.

Keywords: Câncer; Laser therapy; Oncology; Radiodermatitis.

\section{Resumen}

El presente trabajo tiene como objetivo evaluar la efectividad del tratamiento con terapia láser en pacientes que han desarrollado dermatitis inducida por radiación. Para lograr el objetivo propuesto y utilizando la revisión integradora de la literatura con un enfoque cualitativo como ruta metodológica, se realizó una búsqueda en las bases de datos SCIELO, PUBMED, LILACS y BDENF; en idiomas: portugués, inglés y español, entre los años 2010 a 2020. En el $86 \%$ de las encuestas seleccionadas para redactar este artículo, se obtuvieron resultados positivos en cuanto a la efectividad del tratamiento con terapia láser, promoviendo la reparación tisular debido a la reducción de la inflamación. / dolor e inducción de la síntesis de colágeno a dosis adecuadas. Sin embargo, otro estudio señaló una buena eficacia con la PDL, pero observó reacciones transitorias y posteriores al tratamiento con láser como: dolor, púrpura y eritema. Otros autores concluyeron que la aplicación de LED en pacientes antes de la radioterapia mostró una mejora en la intensidad del dolor debido a la toxicidad cutánea inducida por la radiación. Se concluye que la terapia con láser ha mostrado resultados satisfactorios en el tratamiento de las lesiones cancerosas, constituyendo una valiosa alternativa en la lucha contra la radiodermatitis.

Palabras clave: Cáncer; Oncología; Radiodermatitis; Terapia con láser.

\section{Introdução}

Câncer é um termo que inclui mais de 100 doenças desencadeadas a partir de uma mutação genética no ácido desoxirribonucleico (DNA), cujo as células atípicas se multiplicam de forma desordenada. Essas células invadem tecidos e órgãos de modo incontrolável e agressivo, formando assim os tumores (INCA, 2020). As neoplasias são consideradas um dos principais agravos responsáveis pelo extenso número de óbitos mundialmente, permanecendo somente atrás das notificações de doenças do sistema cardiovascular (Rodrigues et al., 2020).

Embora ocorra uma distribuição homogênea de cânceres entre homens e mulheres, cada tipo de neoplasia tem uma incidência distinta entre os sexos. De acordo com o Instituto Nacional de Câncer (INCA) com referência a 2020, câncer de próstata $(29,2 \%)$, colorretal $(9,1 \%)$ e câncer de traqueia, brônquios e pulmão $(7,9 \%)$ afetaram principalmente os homens, enquanto as mulheres foram acometidas principalmente por câncer de mama (29,7\%), seguido pelo colorretal $(9,2 \%)$ e o câncer de colo do útero $(7,5 \%)$.

Os fatores de risco para uma elevada prevalência das neoplasias, estão ligados principalmente à fatores individuais e ambientais, a exemplo da hereditariedade, exposição a substâncias nocivas, desequilíbrio hormonal, como também ao estilo de vida do indivíduo: prática de exercícios, dieta, sedentarismo, estão diretamente ligados (Mentella et al., 2019). Singularmente, dentre os cinco e mais importantes contribuintes para o desenvolvimento dos cânceres, têm-se a baixa ingesta de frutas e vegetais, alto índice de massa corpórea, ausência de atividade física, o tabagismo e o consumo de álcool, somado com algumas infecções crônicas (Barchitta et al., 2018; Ricceri et al., 2017; Hodge et al., 2016; Bodén et al., 2019).

O processo de carcinogênese - exposição das células aos agentes carcinógenos - é dividido em três estágios: iniciação, promoção e progressão, respectivamente. O último estágio é caracterizado pela multiplicação descontrolada, ou seja, as células alteradas tornam-se incapacitadas de retornar ao estágio anterior. Dessa forma, surgem as primeiras manifestações clínicas da doença e o paciente entra em contato com o plano terapêutico que, em grande parte, será através de uma quimioterapia ou radioterapia (INCA, 2020).

As terapêuticas possuem atividade curativa, adjuvante ou paliativa. A radioterapia encontra-se dentro de alguns subgrupos dessas terapêuticas, é um tratamento considerado seguro e eficaz contra neoplasias, sua ação consiste em emitir 
radiação ionizante em uma determinada parte do corpo, visando destruir e impedir que ocorra um aumento nas células tumorais. Embora seja considerada eficaz na redução dos tumores, causa na maioria dos pacientes diversos efeitos adversos (Ruysscher et al., 2019).

Considerado um dos principais efeitos adversos, as radiodermatites - inflamação causada pela exposição secundária da pele à radiação ionizante - prejudica a capacidade das células basais de se proliferarem rapidamente, afetando o processo de cicatrização (Bray, Simmon, Wolfson \& Nouri, 2016). Essas lesões decorrentes do tratamento em questão se manifestam de diferentes formas, a depender da quantidade de radiação absorvida, podem desencadear um simples eritema (com ou sem prurido), descamação (seca ou úmida), dor, alteração da imagem corporal e até necrose tecidual (Rodrigues et al., 2020; Lopes et al., 2019).

Para classificar o grau de evolução da radiodermatite utilizam-se escalas para avaliar a pele, sendo as mais utilizadas a Radiation Therapy Oncology Group (RTOG) da European Organisation for Research and Treatment of Cancer, e a da Common Terminology Criteria for Adverse Events (CTCAE) da National Cancer Institute, esses graus podem variar de zero a cinco em ordem crescente de toxicidade (Yee et al., 2018).

Há tratamentos disponíveis no mercado para as radiodermatites, uns mais efetivos que outros, do ponto de vista médico. Muito se é discutido sobre fármacos tópicos a base de produtos naturais tanto para a prevenção quanto para o tratamento das dermatites consequentes da radiação. Nesta revisão integrativa iremos discutir sobre um instrumento terapêutico presente no modelo biomédico de assistência desde 1960 - a laserterapia - e apontar os principais benefícios no processo de cicatrização e cura das lesões citadas (Rocha et al., 2018; Rossi et al., 2017).

A terapia com laser tem sido considerada um recurso de grande valia em virtude da estimulação no tecido por meio da ampliação da luz por emissão estimulada de radiação sobre as lesões, favorecendo um processo cicatricial ao elevar a multiplicação das células reparativas, bem como reestruturar o colágeno. É uma terapêutica que envolve menor custo e promove melhora nos processos cicatriciais (Lima et al., 2018; Araújo \& Martins, 2019; Andrade, Lima \& Albuquerque, 2010; Salomé \& Ferreira, 2017).

Diante desses pressupostos é pertinente estudar os efeitos que a laserterapia proporciona no tratamento das radiodermatites em pacientes oncológicos, analisando assim sua eficácia, somado a isso, a escassez de pesquisas voltadas a essa temática justifica a elaboração deste artigo como meio de levantar e divulgar esta terapêutica para os profissionais de saúde, melhorando cada vez mais a assistência fornecida para pacientes portadores de cânceres. Após contextualizar a problemática, algumas indagações acerca da proposta do estudo nos remetem a refletir a seguinte questão norteadora: pacientes portadores de radiodermatites quando submetidos a laserterapia demonstram uma melhora clínica? Nessa perspectiva, a presente pesquisa tem como objetivo analisar evidências do uso e eficácia da terapia a laser, no tratamento de pacientes que desenvolveram dermatites induzidas pela radiação.

\section{Metodologia}

Trata-se de uma revisão integrativa da literatura, de natureza qualitativa. De acordo com Pereira et al. (2018), os aspectos metodológicos da pesquisa qualitativa permitem ao pesquisador emitir opiniões próprias sobre o fenômeno de estudo interpretado.

Para construir a questão norteadora utilizou-se a estratégia PICO (População, Intervenção, Controle e Resultados), conduzida de acordo com as seguintes etapas de elaboração: escolha do tema, formulação da questão norteadora, busca na literatura, coleta de dados, seleção e análise crítica dos estudos, discussão dos resultados e apresentação da revisão. 


\section{Critérios de elegibilidade e estratégia de busca}

Após determinar o objetivo, formular hipóteses a serem analisadas, foi realizada a busca de material científico em bases de dados informatizadas para identificar e coletar o máximo de pesquisas relevantes sobre o tema a ser discutido. A busca foi executada no período de julho a setembro de 2020 utilizando as bases de dados relacionadas à área da saúde: Eletronic Library Online (SCIELO), Public Medline (PUBMED), Literatura Latino Americana do Caribe em Ciências da Saúde (LILACS) e Base de Dados da Enfermagem (BDENF).

Os descritores incluídos para pesquisa em todas as bases de dados foram: "Radiodermatite", "Terapia com Luz de Baixa Intensidade", "Radioterapia" e "Dermatopatias", previstos nos Descritores de Ciências em Ciências da Saúde (DeCS). O cruzamento desses termos ocorreu a partir do operador booleano AND e OR.

Para seleção dos artigos, foram utilizados os seguintes critérios de inclusão: estudos disponibilizados na íntegra, em português, espanhol ou inglês que respondessem à pergunta de investigação desta pesquisa e que correspondessem ao recorte temporal de 10 anos (2010-2020). Foram aplicados os critérios de exclusão: publicações no formato de carta editorial, tese e dissertação, pôster ou artigos que não contemplassem sobre o uso do laser para o tratamento de radiodermatites.

Posteriormente organizou-se uma lista com os títulos dos artigos encontrados através de uma planilha do software Microsoft Excel by Office 2019, sendo realizada manualmente a identificação e exclusão dos arquivos em duplicatas. A seleção mediante critérios de inclusão e exclusão foi feita por dois revisores, de forma independente. O parecer de um terceiro autor foi solicitado nos estudos que geraram alguma discordância.

Após análise do título, resumo e leitura na íntegra, foram retiradas as seguintes informações: autores, ano de publicação, título e os principais resultados apresentados nas pesquisas.

\section{Análise dos dados}

Os resultados foram apresentados por meio de análise descritiva e exploratória dos dados, evidenciando a relação / contribuições da simulação entre os estudantes de enfermagem, professores e enfermeiros.

\section{Resultados e Discussão}

Diante da triagem inicial realizada nas bases de dados, foram encontrados 2.238 estudos, desse total apenas 1.538 foram documentos publicados nos últimos dez anos. Após busca, excluiu-se 218 publicações indexadas de forma simultânea em duas ou mais bases de dados. Em sequência, realizou-se a leitura criteriosa dos títulos e dos resumos que resultaram na seleção de 39 artigos para leitura na íntegra. A amostra final desse estudo foi composta por seis artigos indexados nas bases de dados propostas (Figura 1). 
Figura 1 - Fluxograma dos estudos incluídos.

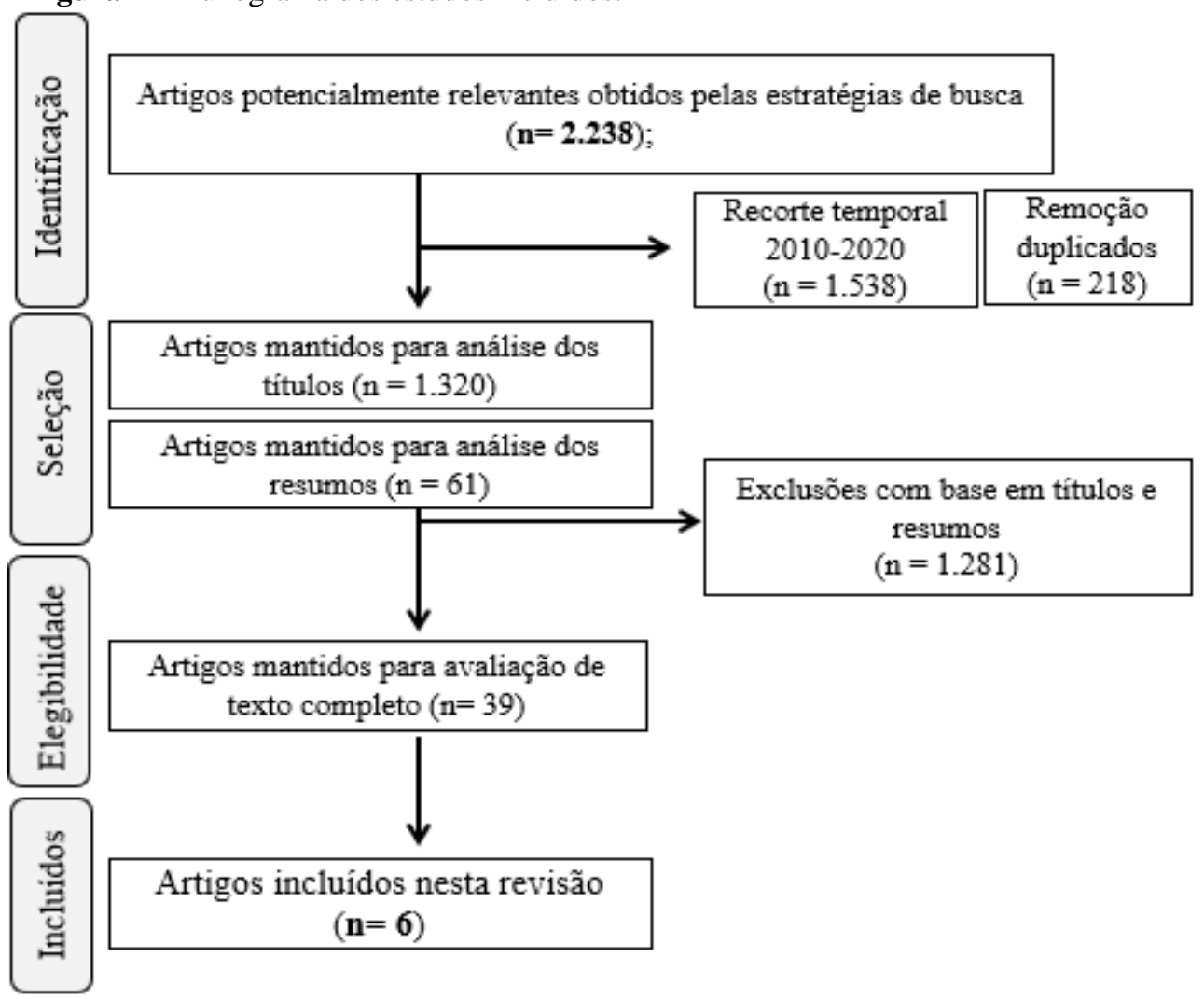

Fonte: Autores (2020).

Os artigos incluídos para compor o presente artigo foram publicados entre o período de 2013 a 2020, com uma publicação em cada ano. Não sendo incluídos artigos nos anos de 2015 e 2016. Quanto ao tipo de estudo dessas pesquisas, três artigos utilizaram a revisão integrativa da literatura (50\%), seguidos da revisão sistemática, estudo prospectivo e ensaio clínico randomizado que contabilizou cada um $(16,7 \%)$.

Na Tabela 1 nota-se as principais características dos artigos selecionados que enfatizam a eficácia da laserterapia. Esse tratamento promove uma recuperação clínica significativa nas radiodermatites, incluindo a redução da dor e inflamação, bem como promove uma melhora do processo cicatricial, trazendo menos riscos no prognóstico do cliente. 
Tabela 1 - Caracterização dos estudos incluídos.

AUTORES/ANO

RODRIGUES et al., 2020

LOPES, SANTOS \& GONÇALVES, 2019

ROCHA et al., 2018

ROSSI et al., 2017

COSTA et al., 2014

COSTA et al., 2013

\section{TITULO}

Uso do laser de baixa intensidade nas radiodermatites: revisão sistemática.

Efeitos do laser de baixa potência em radiodermatites de pacientes com câncer mamário: uma revisão de literatura.

Evidências científicas sobre os fatore associados às qualidades de vida de pacientes com radiodermatite.

Effect of laser therapy on quality of life in patients with radiation-induced breast telangiectasias.

Phototherapy $660 \mathrm{~nm}$ for the prevention of radiodermatitis in breast cancer patients receiving radiation therapy: study protoco for a randomized controlled trial.

Os benefícios do laser de baixa potência na oncologia.

\section{RESULTADOS}

Em $86 \%$ dos estudos presentes neste artigo, pode-se concluir que o laser de baixa intensidade trouxe benefícios para o estado clínico do paciente, dentre eles, redução da dor (43\%), diminuição considerável no tempo de duração e gravidade da mucosite $(29 \%)$, redução do processo inflamatório e cicatricial das lesões (29\%).

Nos 3 artigos elegidos, observou-se que os LASER'S de baixa potência possuem uma efetiva ação no reparo e na regeneração tecidual das lesões cutâneas ocasionadas pelos efeitos adversos da radioterapia, por outro lado, o LASER com gás de InGaAlP, não proporcionou importante redução no surgimento de radiodermatite superior ou igual a grau 2, como também não diminuiu o nível de dor secundária a esta lesão.

O uso de abordagens como a laserterapia, o óleo de emu e a radioterapia modulada por intensidade representaram as principais intervenções que proporcionam uma melhor qualidade de vida por trazerem impactos positivos na redução da dor e prurido, melhora na imagem corporal e redução nas crises de ansiedade e depressão.

Pacientes mostraram significância estatística melhorias no Skindex-16, emocional e funcional. As pontuações do Breast-Q também melhoraram: diminuição em aspectos físicos e cosméticos específicos preocupações comuns à pele irradiada da mama. Eventos adversos comuns: dor transitória pós-tratamento e vermelhidão.

A aplicação de laser de baixa potência demonstrou ser uma opção de tratamento modelo para a prevenção da radiodermatite ao apresentar resultados positivos na reparação tecidual de lesões, bem como diminuição da inflamação e indução da síntese de colágeno. Somado a isso, não houve associação entre o uso dessa terapêutica com o surgimento de eventos adversos e é custo-efetiva.

Foram selecionados 39 artigos por apresentarem evidências científicas relevantes sobre os benefícios do uso da laserterapia na área oncologia. 
No tocante a análise das características dos resultados, é possível observar uma convergência entre as ideias dos autores, percebe-se que a laserterapia é uma abordagem não invasiva e que apresenta baixo custo, além de possuir resultados positivos tanto no tratamento quanto na reabilitação/recuperação das radiodermatites.

Alguns métodos podem ser indicados para ajudar na cura ou melhora dessas lesões, um desses é a laserterapia, intervenção que apresentou resultados significantes na resolução dos processos inflamatórios, redução da dor, formação de edemas e preservação de tecidos e nervos circunvizinhos. Um estudo recomenda que para evitar agravos teciduais deve ser utilizada uma dose inferior a 7J, ondas entre 600 e $1000 \mathrm{~nm}$ e potência de $1 \mathrm{mw}$ e $5 \mathrm{w} / \mathrm{cm} 2$. Ademais, assegura não ser responsável por acelerar a proliferação de células cancerígenas (Costa et al., 2014).

Uma revisão sistemática apontou diferentes tipos de laser no tratamento das lesões, dentre eles: Laser Corante de Pulso Longo (LPDL), Laser de Luz Intensa Pulsada (IPL), Laser Pulsado de Contraste (PDL), Laser de Baixa Intensidade (LBI), Ligh-Emitting Diode (LED) e FotoBioModulação (FBM). Vale salientar que, as diferentes classificações possuem benefícios e complicações específicas (não encontradas claramente na literatura científica após comprovação clínica). No entanto, a pesquisa bibliográfica fez uma comparação entre dois métodos (LPDL e IPL), assim, observou maior eficácia no LPDL (Rodrigues et al., 2020).

Entretanto, outro estudo apontou como dose da terapia a laser 2 a $3 \mathrm{~J} / \mathrm{cm}$ que foi aplicado diariamente, ou no mínimo de três vezes na semana, sem resultar em nenhum efeito adverso, nesse mesmo estudo os 230 pacientes analisados, receberam uma dose de 1 a $2 \mathrm{~J} / \mathrm{cm}$ por ponto aplicado, para o tratamento do linfedema, tendo como resultado a redução do volume do membro (Lopes et al., 2019; Costa et al., 2014).

Ademais, em uma pesquisa foi relatado à indução de danos e comprometimento funcional das células do epitélio da mucosa oral e das estruturas glandulares salivares que resultou em uma mucosa frágil e fluxo salivar reduzido, porém tanto a dor como a gravidade da lesão foi reduzida significativamente com a utilização da laserterapia e, segundo os autores, a terapia a laser mostrou-se eficaz tanto para a prevenção quanto para o tratamento das lesões bucais resultando na diminuição das dores e melhora da capacidade de ingestão (Costa et al., 2013).

Em uma segunda revisão sistemática, com análise de sete artigos - um estudo prospectivo duplo-cego randomizado, caso-controle e revisão sistemática - concluíram que em $86 \%$ das pesquisas houveram resultados positivos em relação à eficácia do tratamento. Os resultados demonstraram reparação tecidual devido à redução da inflamação/dor e à indução da síntese de colágeno quando usados nas doses corretas, um dos estudos citou a utilização da fotomodulação com diodos emissores de luz (LEDs), administradas imediatamente após a terapia de radiação em pacientes com câncer de mama, a fim de reduzir a incidência de reações cutâneas, segundo a Classificação do Cancer Institute (NCI) 1, 2 ou 3. Já um segundo estudo mostrou boa eficácia com o PDL, entretanto observou reações transitórias e do tratamento pós-laser como: dor, púrpura e eritema. Outros autores concluíram que aplicação do LED em pacientes antes da radioterapia apresentou melhoria na intensidade da dor devido à toxicidade da pele induzida por radiação (Rodrigues et al., 2020).

Em contrapartida, outro artigo constatou alguns prejuízos para os pacientes, como fatores que comprometem a qualidade de vida (dor, desconforto, fadiga, reações emocionais, alteração da sensibilidade, da imagem corporal e do sono). Nesse mesmo estudo ainda foi possível notar que a laserterapia, a aplicação tópica do óleo de emu e a radioterapia modulada por intensidade, em conjunto, demonstram ser eficazes para o paciente em uma visão ampla, além da lesão em um aspecto biopatológico (Rocha et al., 2018).

Por fim, percebe-se que o uso do laser é indicado como uma intervenção terapêutica eficaz para as radiodermatites na maioria dos artigos apresentados e discutidos acima, apesar de existirem contrapartidas quando analisado o aspecto biopsicossocial. 


\section{Considerações Finais}

Esta revisão integrativa identificou que a laserterapia demostrou resultados satisfatórios no processo de reparo tecidual com efeitos duradouros, constituindo uma alternativa valiosa no combate as radiodermatites. Percebe-se que a maioria dos estudos apontam os benefícios e a eficácia da terapia. No entanto, alguns malefícios são importantes ressaltar como: dor, desconforto, fadiga, reações emocionais, alteração da sensibilidade, da imagem corporal e do sono.

Pode-se concluir que a terapia a laser é uma boa possibilidade de tratamento para lesões oncológicas (induzidas pela radiação), desde que seja observado um controle de qualidade visando potencializar as propriedades terapêuticas e minimizando os riscos de infecções e baixa qualidade de vida.

Em virtude dos fatos mencionados, mostra-se a relevância de novos trabalhos voltados a temática. Consequentemente, sugere-se a ampliação de futuras pesquisas referentes a essa temática no intuito de agregar mais evidências relacionadas, como também qualificar os benefícios proporcionados para o cliente ao utilizá-la como tratamento para as radiodermatites, podendo assim impactar positivamente no processo saúde-doença e melhorar sua qualidade de vida.

\section{Referências}

Andrade, A. G., Lima, C. F., \& Albuquerque, A. K. B. (2010). Efeitos do laser terapêutico no processo de cicatrização das queimaduras: uma revisão bibliográfica. Revista Brasileira de Queimaduras, 9(1), 21-30.

Andrade, F. D. S. D. S. D., Clark, R. M. D. O., \& Ferreira, M. L. (2014). Effects of low-level laser therapy on wound healing. Revista do Colégio Brasileiro de Cirurgiões, 41(2), 129-133.

Araújo, M. J. S., \& Martins, G. B. (2019). Utilização do diodo emissor de luz (led) na cicatrização de queimaduras: revisão sistemática da literatura. Revista Pesquisa em Fisioterapia, 9(1), 108-119.

Barchitta, M., Maugeri, A., Quattrocchi, A., Agrifoglio, O., Scalisi, A. \& Agodi, A. (2018). The Association of Dietary Patterns with High-Risk Human Papillomavirus Infection and Cervical Cancer: A Cross-Sectional Study in Italy. Nutrients, 10(10.3390), 1-12.

Bodén, S., Myte, R., Wennberg, M., Harlid, S., Johansson, I., Shivappa, N., \& Nilsson, L. M. (2019). The inflammatory potential of diet in determining cancer risk; A prospective investigation of two dietary pattern scores. PLOS ONE, 14(4), 1-16.

Bray, F. N., Simmons, B. J., Wolfson, A. H., \& Nouri, K. (2016). Acute and Chronic Cutaneous Reactions to Ionizing Radiation Therapy. Dermatol Ther (Heidelb), 6(2), 185-206.

Costa, M. M., Silva, S. B., Quinto, A. L. P., Pasquinelli, P. F. S., dos Santos, V. D. Q., de Cássia Santos, G., \& Veiga, D. F. (2014). Phototherapy 660 nm for the prevention of radiodermatitis in breast cancer patients receiving radiation therapy: study protocol for a randomized controlled trial. Trials, $15(1), 1-6$.

Costa, R. O., Catão, M. H. C. V., Costa, I. R. R. S., Silva, A. D. L., \& Pires, E. G. (2013). Os benefícios do laser de baixa potência na oncologia. Revista Brasileira de Ciências e Saúde (RBCS), 11(37), 67-72.

Hodge, A. M., Basset, J. K., Shivappa, N., Hebert, J. R., Inglês, D. R., Giles, G. G. \& Severi, G. (2016). Dietary Inflammatory Index, Mediterranean Diet Score e Lung Cancer: A Prospective Study. Cancer Causes Control, 27(7), 907-917.

INCA (2020). Câncer: O que é câncer? https://www.inca.gov.br/numeros-de-cancer.

Lima, N. E. P., Gomes, G. M., Feitosa, A. N. A., Bezerra, A. L. D., \& Sousa, M. N. A. (2018). Laserterapia de baixa intensidade no tratamento de feridas e a atuação de enfermagem. Revista Enfermagem UFPI, 7(1), 50-56.

Lopes, A. E. V., Santos, D. T., \& Gonçalves, G. D. (2019). Efeitos do laser de baixa potência em radiodermatites de pacientes com câncer mamário: uma revisão de literatura.

Mentella, M. C., Scaldaferri, F., Caterina, R., Gasbarrini, A., \& Miggiano, G. A. D. (2019). Câncer e dieta mediterrânea: uma revisão. Nutrients, 11(9), 1-25.

Pereira A.NS. et al. (2018). Metodologia da pesquisa científica. UAB/NTE/UFSM. https://repositorio.ufsm.br/bitstream/handle/1/15824/Lic_Co mputacao_Metodologia-Pesquisa-Cientifica.pdf?sequence=1.

Ricceri, F., Giraudo, M. T., Fasanelli, F., Milanese, D., Sciannameo, V., Fiorini, L., \& Sacerdote, C. (2017). Diet and endometrial cancer: a focus on the role of fruit and vegetable intake, Mediterranean diet and dietary inflammatory index in the endometrial cancer risk. BMC Cancer, $17(1), 757$.

Rocha, D. M., Pedrosa, A. O., Oliveira, A. C., Bezerra, S. M. G., Benício, C. D. A. V., \& Nogueira, L. T. (2018). Evidências científicas sobre os fatores associados à qualidade de vida de pacientes com radiodermatite. Revista Gaúcha de Enfermagem, 39, e2017-0224.

Rodrigues, J. M. S., da Silva Acosta, A., Gouvea, P. B., \& Massaroli, R. (2020). Uso do laser de baixa intensidade nas radiodermites: revisão sistemática/Low intensity laser use in radiotherapy lesions: systematic review. Journal of Nursing and Health, 10(2), 1-14. 
Research, Society and Development, v. 10, n. 2, e17810212364, 2021

(CC BY 4.0) | ISSN 2525-3409 | DOI: http://dx.doi.org/10.33448/rsd-v10i2.12364

Rossi, A. M., Blank, N. R., Nehal, K., Dusza, S., \& Lee, E. H. (2018). Effect of laser therapy on quality of life in patients with radiation-induced breast telangiectasias. Lasers in surgery and medicine, 50(4), 284-290.

Ruysscher, D., Niedermann, G., Burnet, N. G., Siva, S., Lee, A. W. M., \& Hegi-Johnson, F. (2019). Radiotherapy toxicity. Nature Reviews Disease Primers, $5(1), 13$.

Salomé, G. M., \& Ferreira, L. M. (2017). Low intensity laser and microcurrent in scratching of skin wounds in rats. Journal of Nursing UFPE on line, 11(9), 3567-3573.

Wong, R. K., Bensadoun, R. J., Boers-Doets, C. B., Bryce, J., Chan, A., Epstein, J. B., ... \& Lacouture, M. E. (2013). Diretrizes de prática clínica para prevenção e tratamento de reações de radiação agudas e tardias do MASCC Skin Toxicity Study Group. Supportive Care in Cancer, 21(10), 2933-2948.

Yee, C., Wang, K., Asthana, R., Drost, L., Lam, H., Lee, J., Danny, V. \& Chow, E. (2018). Radiation-induced skin toxicity in breast cancer patients: A systematic review of randomized trials. Clin Breast Cancer, 18(5), 825-840. 